\title{
Antiepileptic drugs and suicidality
}

This article was published in the following Dove Press journal:

Drug, Healthcare and Patient Safety

25 September 2010

Number of times this article has been viewed

\section{Jeffery W Britton' \\ Jerry J Shih ${ }^{2}$}

'Mayo Clinic, Rochester, MN, USA;

${ }^{2}$ Mayo Clinic, Jacksonville, FL, USA

Correspondence: Jeffrey W Britton

Mayo Clinic Dept of Neurology, 200

Ist St SW, Rochester, MN 55905, USA

$\mathrm{Ph}+\mathrm{I} 5075381036$

Fax +l 5072664419

Email britton.jeffrey@mayo.edu
Abstract: The risk of suicide in patients with epilepsy is significantly higher than the general population. There are many hypotheses as to the reasons for this, but the potential role of antiepileptic drugs (AEDs) in increasing suicidality has recently been brought into question. In 2008, the U.S. Food and Drug Administration (FDA) published a warning after a meta-analysis of data from all clinical trials involving AEDs found a suicidality risk of 0.43 per 1000 patients in active drug arms of these clinical trials compared to a rate in the placebo arm of 0.22 . While an increased risk for individual AEDs was found in two, the FDA decided to issue a warning for the entire AED class. While this decision and the meta-analysis findings have been considered controversial, and have created concern that this stated risk may dissuade use of AEDs by patients who would benefit from them, it has led to increased awareness of the risk of suicidality and psychiatric co-morbidity in this patient group. In this article, the association of epilepsy and AEDs with psychiatric disease and suicidality are reviewed, perspective as to the significance and limitations of the FDA's findings are discussed, and some options for suicidality screening and their potential utility in clinical care are evaluated.

Keywords: antiepileptic drugs, suicidality, FDA, behavior

Suicide is a tragic outcome for patients with epilepsy given the therapeutic advances achieved in recent decades. Yet, suicide accounts for up to $11 \%$ of all deaths in epilepsy patients, which is significantly higher than would be predicted by the United States general population suicide mortality rate of $1.4 \%$ and incidence rate of 11.6 per 100,000 people..$^{1-5}$ The rate of suicide as the cause of death in the epilepsy population varies widely, ranging from $0.7 \%-24 \%$ in the literature when limiting review to studies reporting a minimum of 100 deaths. ${ }^{2,3,6-10}$ The reasons for increased suicidality in the epilepsy population are likely numerous given its disruptive effect on socioeconomic factors, quality of life, and central nervous system physiology. ${ }^{11-15}$ However, some researchers have speculated whether antiepileptic drug (AED) treatment contributes to psychiatric comorbidity and suicidality in epilepsy. ${ }^{16,17}$ Indeed, negative quality of life factors and increased measures of distress have been identified in epilepsy patients on AED treatment, even in those with controlled seizures, which in some cases may prompt consideration of discontinuing therapy. ${ }^{12,18-20}$ The concern regarding the role played by AEDs in suicidality was recently heightened by the 2008 United States Food and Drug Administration (FDA) warning of an increased risk of suicidal behavior associated with the AED drug class based on a meta-analysis of all published AED clinical trials. ${ }^{21}$ This announcement prompted a renewed focus on the neuropsychiatric aspects of epilepsy, and raised new concerns about the safety profile of AED treatment. 
In this review, we examine the association between AEDs and epilepsy with mental illness and suicidal behavior, and the data underlying the FDA warning. Screening tools available for clinical use in the evaluation of suicidality and depression are discussed. Finally, a perspective regarding the FDA findings as it relates to the management and counseling of AED therapy in epilepsy patients is provided.

\section{Psychiatric and suicidal comorbidity in epilepsy: epidemiological considerations Epilepsy epidemiology and effects of treatment}

The age-adjusted incidence of epilepsy in the Olmsted County population has been determined to be 44 per 100,000 people, with a cumulative incidence of 3 per 100 through to the age of 75 years. The age-adjusted prevalence of active epilepsy is 6.8 per 1,000 people. ${ }^{22}$ Applied to an estimated US population of 350 million, this equates to 2.4 million patients in the US with epilepsy. AEDs are the mainstay of epilepsy treatment, and are associated with a 60\%-70\% efficacy rate. ${ }^{23}$ Nonmedication therapies include surgery, ketogenic diet, and neurostimulation therapies. However, surgical treatment is only appropriate for a select proportion of epilepsy patients and many epilepsy surgery patients still require AED therapy despite an excellent outcome. Currently available neurostimulation therapies in place and under development are palliative in most cases, and the ketogenic diet has not proven to be feasible for widespread use, at least in adult patients. Inadequate seizure control has been associated with injury, and injury can occur upon AED withdrawal even when performed in a controlled setting. ${ }^{24,25}$ In addition, poor seizure control has been shown to contribute to higher mortality in epilepsy, and is a consistent risk factor in sudden unexplained death in epilepsy patients (SUDEP). ${ }^{26,27}$ Thus, AEDs are a critical tool for clinicians in epilepsy management.

\section{Epilepsy and mental illness}

Psychiatric comorbidity in epilepsy is well established. The prevalence of depression in epilepsy patients ranges from $20 \%-55 \%{ }^{28}$ and psychosis has been reported in $6 \%-9 \%{ }^{29}$ These rates are significantly higher than those seen in the general population. Anxiety disorders and suicidal ideation have also been found to be increased in patients with epilepsy. ${ }^{30}$ However, not all psychiatric disorders have an overrepresentation in the epilepsy population, for example, bipolar affective disorder. ${ }^{31}$
Mental illness is likely underrecognized and undertreated in the epilepsy population. In some cases this results from an overlap between the symptoms and signs of both conditions. During the typical office visit with the epilepsy patient, clinicians often focus on assessing seizure control and determining medication adjustments, frequently at the expense of the evaluation of mental and psychosocial health. Neurology and epilepsy training programs lack emphasis on the evaluation and treatment of mental illness, contributing to an underrecognition of these disorders in this population. ${ }^{32}$ In addition, some epilepsy patients may underreport or minimize symptoms of depression and anxiety. Epilepsy patients have variable and inconsistent access to psychiatric services, and those who do are often undertreated for fear of exacerbating seizure activity. ${ }^{28}$

Epilepsy appears to have a higher association with depression when compared to other chronic nonneurologic conditions. ${ }^{33}$ This epilepsy-depression association is currently thought to be secondary to a shared neurobiological basis as opposed to depression resulting from the psychosocial limitations imposed by recurrent seizures. However, this subject remains controversial. ${ }^{28,34}$ Some investigators have shown a higher prevalence of depression with epilepsy compared to other chronic neurologic disorders, supporting the concept of seizures and epilepsy both arising from the same substrate of CNS dysfunction. ${ }^{35}$ However, others have not shown a difference in the rate of depression in epilepsy and other disabling noncentral nervous system (CNS) neurological disorders. ${ }^{33}$

\section{AEDs and mental illness}

The possible role of AEDs in the development of depression in epilepsy has been the subject of controversy for many years. Some researchers have shown an association between mental illnesses and AED use. ${ }^{36}$ However, in many studies on this topic researchers have not been able to fully account for confounding factors such as preexisting psychiatric history and family history of mental illness. ${ }^{37,38}$ Some have shown incomplete seizure control to be associated with a higher rate of psychiatric comorbidity, arguing indirectly for a potential favorable role of AEDs if their use were to lead to maintenance of seizure freedom. Jacoby et al used the Hospital Anxiety and Depression Scale to demonstrate depression in $21 \%$ of 168 epilepsy patients. ${ }^{39}$ The depression rate in this study was shown to have the highest correlation with measures of current seizure activity. O'Donoghue et al compared the rates of depression and anxiety in epilepsy patients who were well-controlled and those with continuing 
seizures. The rate of depression was $33 \%$ in patients with more than one seizure per month, $11 \%$ in those with less than one per month, and $6 \%$ in those controlled on medications. ${ }^{40}$ However others have found evidence for psychopathology during periods of seizure control, raising concerns about the effects of "forced normalization", in turn raising questions about whether there is always a direct relationship between seizure control and psychiatric status. ${ }^{41}$

\section{Epilepsy and suicide risk}

The suicide rate in patients with epilepsy is higher than in the general population. The standardized mortality ratios (SMR) of suicide in epilepsy compared to the general population range from 3.5 to $5.8 .^{42,43}$ Research evaluating the proportionate mortality ratios (PMR) of suicide have shown suicide to account for $1 \%$ to $33 \%$ (mean $=11 \%$ ) of all deaths in the epilepsy population, which is significantly higher than the $1.1 \%-1.2 \%$ rate in the general population. ${ }^{3}$ Even though many researchers have shown an increased suicide rate in the epilepsy population, in some population-based studies an increased incidence was not found. ${ }^{44,45}$ However, since accidental or seizure-related motor vehicle accidents, falls or drowning, and accidental overdose in epilepsy patients cannot always be distinguished from intentional events, it is possible that the epilepsy suicide rate documented in the literature is an underestimate.

Risk factors for suicide in epilepsy include age of seizure onset prior to age 18 years, the presence of preexisting mental illness, and co-treatment with antipsychotic therapy. ${ }^{46}$ Although suicide is more common in epilepsy in the setting of coexistent psychiatric illness, epilepsy has also been found to be an independent risk factor for suicide, showing a calculated SMR of 1.99 in a group of epilepsy patients without presuicide evidence of mental illness. ${ }^{47}$ In contrast to the general population, male gender is not a consistent risk factor in epilepsy suicidality. ${ }^{48,49}$ Polytherapy, poor seizure control, and seizure localization or type have also not been determined to be consistent risk factors. ${ }^{3,46}$ The duration of the epilepsy also does not directly correlate with suicide risk; in fact, in one study, the highest suicide risk was observed in the first six months following diagnosis. ${ }^{47}$ It is also important to note that suicide may occur as an unpredictable acute phenomenon in association with periictal psychosis. ${ }^{50}$ Interestingly, suicidality has also been implicated as a risk factor for the eventual development of epilepsy, arguing again for the concept of mental illness and epilepsy both arising from a shared underlying neuropathologic substrate. ${ }^{51}$

\section{AEDs and suicidality risk}

In response to reported cases of suicidal behavior in patients taking AEDs, in 2005 the FDA ordered manufacturers of drugs classified as "anticonvulsants" to report all suiciderelated events in their controlled trial databases. ${ }^{52,53}$ This meta-analysis included data from 199 randomized clinical trials involving 11 anticonvulsant agents, and showed a near doubling of the risk of suicidal behavior compared to placebo when clinical trials for all indications were reviewed (odds ratio [OR], 1.8; 95\% confidence interval [CI]: 1.2 to 2.72). Data were available from over 27,800 patients receiving antiepileptic drugs and over 16,000 patients on placebo. Suicide behavior was defined as suicidal ideation, preparatory acts, suicide attempts, and successful suicidal action. Suicide behavior occurred in 4.3 per 1,000 patients treated with antiepileptic drugs in the active arm compared with 2.2 per 1,000 patients in the placebo arm, leading to the public statement that the suicidality rate was double that of placebo. ${ }^{54}$ Of all the suicide behavior reported, suicidal ideation accounted for $67.6 \%$, preparatory acts for $2.8 \%$, suicide attempts for $26.8 \%$, and completed suicides in $2.8 \%{ }^{55}$ This meta-analysis found four completed suicides in the active AED treatment arms of these studies and none in the placebo arms. ${ }^{56}$ Of the four people who successfully committed suicide, two were taking antiepileptic drugs for epilepsy, and two were in psychiatry clinical trials. There were 30 suicide attempts by patients taking active medication, and eight in the placebo arms. The suicide behavior risk was highest for those patients in epilepsy trials versus trials for other indications (OR, 3.5; 95\% CI: 1.3 to 12.1 ).

The FDA issued a warning in January 2008, which can be accessed at: http://www.fda.gov/Drugs/DrugSafety/ PostMarketDrugSafetyInformationforPatientsandProviders/ ucm 100200.htm, and a detailed report was subsequently published. ${ }^{53}$ The lay press emphasized the FDA's statement that anticonvulsants were associated with "about twice the risk of suicidal thoughts", without specifying the absolute risk. Public attention to this was probably sensitized by the similarity of this report to warnings issued a few years previously regarding the increased risk of suicide in children associated with selective serotonin reuptake inhibitor (SSRI) use, and the two fold increased risk of vascular events associated with rofecoxib (Vioxx). The FDA's initial response was to recommend a black box warning for the entire anticonvulsant class, similar to that issued for SSRI's. ${ }^{52}$ Subsequently, after much deliberation, a nonblack box warning was decided, and the FDA required manufacturers of drugs classified as anticonvulsants to prepare 
a patient and provider guideline to assist in communicating this increased risk.

So, do antiepileptic drugs increase the suicide risk in a clinically significant manner? The absolute risk, though nearly double that in the placebo study arms, reflects an overall absolute suicidality rate of $0.43 \%$ compared to $0.22 \%$ with placebo, meaning that for every two patients with suicidal behavior associated with anticonvulsant use, 998 patients would not. Similarly, it should be recognized that of the 27,863 patients enrolled in the active drug arm in these studies, four actual suicides occurred and two of them were by patients in psychiatry trials. The high morbidity, mortality, and noncompliance rates inherent in the treatment of epilepsy have raised additional concerns that any such warning would dissuade some patients from taking medications in which they had a greater chance of helping their condition than of leading to suicide risk.

The FDA opinion was not embraced by practicing epileptologists or the various professional societies devoted to treating patients with epilepsy. ${ }^{32}$ The American Epilepsy Society, the American Academy of Neurology, and the Epilepsy Foundation of America issued statements urging physicians and patients to exercise caution in interpreting the FDA ruling. The validity of the meta-analysis methodology used by the FDA in forming the basis of the recommendation was questioned. ${ }^{54,56}$ There were criticisms that the data on suicide behavior was not gathered in a systematic prospective fashion, and that the presence of suicidality was gleaned from self-reported adverse events, the collection of which varied from site to site. Kanner and Hesdorffer argued that patients on active drugs tend to report more adverse events in general than those on placebo, stating that this phenomenon may increase the chance of other negative symptoms being reported secondary to a "coat-tail effect". The FDA has also been criticized for using a portion of the 199 clinical trials for the main statistical analysis which eliminated trials without suicide behavior in either arm. An ancillary analysis which included all events was performed but was criticized for the lack of calculation of a confidence interval. None of the patients in the felbamate clinical trials reported suicide behavior, and carbamazepine and valproate actually exhibited protective effects, yet these were included in the FDA AED class warning. Regarding the eight other AEDs included in the warning, only lamotrigine and topiramate individually reached statistical significance (see Table 1). ${ }^{53}$ Statistical analysis for heterogeneity across all trials did not produce significant results. Thus, the extrapolation of suicidality risk to the entire class of antiepileptic agents has been criticized.

The FDA approach in issuing a blanket warning encompassing all anticonvulsants is questionable. Despite the above points, the FDA ultimately decided to group all anticonvulsants as a class, and required the same warning for the whole AED class. This approach does not account for scientific data demonstrating different mechanisms of action for each AED. ${ }^{16}$ The FDA's concern that specifically targeting drugs in this FDA warning would adversely prejudice clinical practices against the certain drugs runs counter to past FDA actions. ${ }^{54}$ The use of valproate in women of child-bearing age has decreased after data emerged of the drug's potential for teratogenicity. Felbamate use has significantly decreased since the FDA published its black box warning linking the drug to increased risk for aplastic anemia and fatal hepatotoxicity. The rate of drug escalation for lamotrigine was significantly reduced after data emerged that rapid dose escalation increased the risk for significant skin hypersensitivity reactions. Clinicians make therapeutic decisions daily regarding individual drugs based on that drug's pharmacologic characteristics and risk-benefit ratio. The approach of issuing a warning for the entire class has therefore been criticized.

Even while concern exists about the FDA's approach to this issue, there is no disagreement that initiation of certain AEDs in patients with epilepsy may be associated with increased suicide risk. However, efforts to identify those AEDs with particular increased risk have shown inconsistent results. The ORs ratios of suicidal behavior and specific AEDs found in different studies are summarized in Table 1. In a Danish study in which pharmaceutical claims data were investigated revealed possible differences in suicide risk associated with different AEDs. ${ }^{57}$ The authors reported that AED initiation in general was associated with an increased risk of suicide (OR, 1.84; 95\% CI: 1.36-2.49). In terms of the risk of specific AEDs, clonazepam (OR, 2.01; 95\% CI: 1.25-3.25), valproate (OR, 2.08; 95\% CI: 1.04-4.16), lamotrigine (OR, 3.15; 95\% CI: 1.35-7.34), and phenobarbital (OR, 1.96; 95\% CI: 1.02-3.75) showed increased risk, while the remaining examined AEDs did not. The main weakness in this study was that it did not identify the clinical indication for AED use, and likely included patients treated with AEDs for mood and anxiety disorders, and chronic pain.

In an American cohort study the suicide rate in patients initiating AEDs prescription claims data in a health-system database over a 5 -year period were investigated. ${ }^{58}$ Cox 
Table I Studies in which suicidality potential of specific antiepileptic drugs (AEDs) was investigated

\begin{tabular}{|c|c|c|}
\hline \multirow{2}{*}{$\begin{array}{l}\text { Study } \\
\text { FDA - Levenson } 2008^{53}\end{array}$} & \multicolumn{2}{|c|}{ Specific antiepileptic drugs and main published statistical findings } \\
\hline & Lamotrigine & $2.08(1.03-4.40)$ \\
\hline$N=199$ clinical trials & Topiramate & $2.53(1.21-5.85)$ \\
\hline involving $A E D s$ & All other AEDs & No significant risk \\
\hline Oleson $2010^{57}$ & All AEDs at initiation & $1.84(1.36-2.49)$ \\
\hline All patients prescribed & Clonazepam & $2.01(1.25-3.25)$ \\
\hline AEDs during study pd., & Valproate & $2.08(1.04-4.16)$ \\
\hline \multirow[t]{2}{*}{ all diagnoses } & Lamotrigine & $3.15(1.35-7.34)$ \\
\hline & Phenobarbital & $1.96(1.02-3.25)$ \\
\hline Patorno $2010^{58}$ & Gabapentin & $1.42(1.11-1.80)$ \\
\hline All patients initiated & Lamotrigine & $1.84(1.43-2.37)$ \\
\hline with AED therapy; & Oxcarbazepine & $2.07(1.56-2.80)$ \\
\hline using suicide rate with & Tiagabine & $2.4 \mathrm{I}(1.65-3.52)$ \\
\hline topiramate as reference & Valproate & $1.65(1.25-2.19)$ \\
\hline Gibbons $2010^{59}$ & AED therapy & 13 suicides per 1000 pt-yrs \\
\hline Bipolar affective & No AED therapy & 13 suicides per 1000 pt-yrs \\
\hline disorder population & & No significant difference \\
\hline van Cott $2010^{60}$ & Prior $\mathrm{dx}$ affective disorder & $4.2(2.4-7.5)$ \\
\hline Suicide rates in all pts & AED indication: epil vs other & No significant difference \\
\hline starting AED therapy, & Lamotrigine \& levetiracetam & $10.2(I .1-97.0)$ \\
\hline \multirow[t]{3}{*}{ any indication vs epilepsy } & Valproate & $2.3(1.0-5.3)$ \\
\hline & Phenobarbital, phenytoin, & \\
\hline & carbamazepine & No significant difference \\
\hline Andersohn $2010^{61}$ & "High” depressive potential* & $3.08(1.22-7.77)$ \\
\hline Epilepsy cohort, those & "Low" depressive potential* & $0.87(0.47-1.59)$ \\
\hline with suicidality vs no & Conventional AEDs & $0.74(0.53-1.03)$ \\
\hline diagnosis of suicidality & Barbiturates & $0.66(0.25-1.73)$ \\
\hline
\end{tabular}

Notes: First author, abbreviated summary of study design, and main published statistical findings are shown. * = See text for definition of AEDs defined as having "high" and "low" depressive potential. Results displayed are Odd's ratio + $95 \%$ confidence intervals except where otherwise indicated.

proportional hazards models and propensity score-matched analyses were used to evaluate risk of attempted or completed suicide and combined suicidal acts or violent death, controlling for psychiatric comorbidities and other risk factors as determined by International Statistical Classification of Diseases and Related Health Problems (ICD-9) and current procedural terminology codes. The suicidality rate of several individual AEDs were compared to the rate in patients taking topiramate, and separately carbamazepine, as references. The researchers identified 26 completed suicides, 801 attempted suicides, and 41 violent deaths in 297,620 patients initiating AED therapy. The incidence of the composite outcomes of completed suicides, attempted suicides, and violent deaths in patients receiving AEDs ranged from 6.2 per 1,000 person-years for primidone to 34.3 per 1,000 person-years for oxcarbazepine. The risk of suicidal acts was increased for gabapentin (hazard ratio [HR], 1.42; 95\% CI: 1.11-1.80), lamotrigine (HR, 1.84; 95\% CI: 1.43-2.37), oxcarbazepine (HR, 2.07; 95\% CI: 1.52-2.80), tiagabine (HR, 2.41; 95\% CI: 1.65-3.52), and valproate
(HR, 1.65; 95\% CI: 1.25-2.19), compared with topiramate (see Table 1). As with the Danish study, these study results are confounded by the fact that all patients initiating AEDs were included, not just patients with epilepsy. The fact that gabapentin had the highest suicidality risk is important to note in this respect as it is chiefly used in the treatment of pain, as opposed to epilepsy.

Gibbons et al used the PharMetrics medical claims database to study the relationship between the 11 AEDs identified in the FDA alert, and lithium, to suicide attempts in a cohort of approximately 48,000 patients with bipolar affective disorder. ${ }^{59}$ Suicide attempt rates were compared before and after treatment and with a medication-free control group. Overall, there was no significant difference in suicide attempt rates for patients treated with an AED (13 per 1,000 person-years [PY]) versus no AED or lithium treatment (13 per 1,000 PY). Of note, in patients treated with AEDs, the rate of suicide attempts was significantly higher before treatment ( 72 per 1,000 PY) than after (13 per 1,000 $\mathrm{PY})$. Also, in patients receiving no concomitant treatment 
with an antidepressant, another AED, or antipsychotic, AEDs were shown to have a potential protective effect relative to no treatment (3 per 1,000 PY versus 15 per 1,000 PY). The weakness of this paper, vis-a-vis the context of the present discussion, is that it involved a nonepileptic cohort.

van Cott et al performed a case-control study of elderly American veterans starting AEDs for epilepsy compared to other diagnoses. ${ }^{60}$ Of the 112,096 patients (7,445 with new-onset epilepsy) who were on record as receiving AED monotherapy, 64 individuals had ICD-9 codes indicative of suicide-related behaviors. Rates of suicide-related behaviors were not significantly different for individuals taking AEDs for new-onset epilepsy compared to other diagnoses $(P=0.38)$. While in this study some of the questions raised by the FDA alert were directly examined, the outcome measures were retrospectively measured using ICD-9 data, and not prospectively performed. Of note, this study confirmed previous reports of increased suicidality in epilepsy patients with a previous history of psychiatric disease.

The relative risk of different groups of AEDs for suicidal behavior or self-harm was evaluated using a case-control methodology utilizing data available in the United Kingdom General Practice Research Database. ${ }^{61}$ In this study, based on a cohort of 44,300 epilepsy patients, 453 cases with suicidal behavior or self-harm were matched with 8,962 controls. AEDs were categorized into four subgroups: barbiturates, conventional AEDs, and newer AEDs with "low" or "high" potential for depression. The designation of "low" versus "high" potential for depression was based a priori on whether the frequencies of depressive symptoms in clinical trials of the specific drug in question were lesser or greater than $1 \%$, respectively. The results showed AEDs with "high" depression potential (levetiracetam, tiagabine, topiramate, vigabatrin) were associated with a threefold increased risk of self-harm or suicidal behavior (OR, 3.08; 95\% CI: 1.22-7.77) compared to no AED use within the previous year. The other three AED groups showed no increased risk. Analysis of individual AEDs showed an increase risk for levetiracetam only. In this study, polytherapy was not associated with increased risk.

In summary, there are no prospective randomized controlled studies in the literature to date addressing the question of increased suicidality specifically in epileptic patients starting AEDs, or that allow consistent delineation of those AEDs at particular risk. A prospective study controlling for the many variables that affect suicidality is necessary to more securely implicate specific AEDs with suicide risk.

\section{Suicide risk assessment and diagnostic tools}

Depression, other mental illnesses, and suicidality are not consistently addressed or treated in the course of epilepsy care. In one survey of neurologists, 79\% did not routinely screen for depression in epilepsy patients. ${ }^{28}$ In another performed after the FDA warning, 62\% did not use any scale to routinely screen for depression in persons with epilepsy. ${ }^{32}$ Even for busy clinicians who do routinely assess for mood disorders, screening for suicide risk is challenging as the sensitivity and specificity of current office-based tools are suboptimal. In one study of 4,800 individuals undergoing screening for suicidal ideation, $3.7 \%$ of 803 identified as high risk completed suicide during a 5-year follow-up period, illustrating the nonspecificity of some screening tests for suicidal behavior. ${ }^{62}$ Furthermore, 37 of 67 patients who completed suicides were not identified as at risk by the screening techniques used in this study, illustrating the suboptimal sensitivity of these tools as well.

Several risk factors for suicide are worth keeping in mind when assessing patients. It is important to remember that the majority of patients who commit suicide have an Axis 1 psychiatric diagnosis. Also, those diagnoses of particular importance in the epilepsy population include major depressive disorder, anxiety disorder, and psychosis. For suicide in general, substance abuse is an important risk factor, as are the presence of a specific plan, ownership of a firearm, or access to other means of self-injury and death. ${ }^{5}$ Inquiry as to the presence of these risk factors may help in identifying patients at risk. A few researchers have suggested that the presence of postictal psychosis is a risk factor for suicide in epilepsy. Other risk factors identified in the epilepsy population include a positive family history of suicide, significant financial and family stress, and certain Axis II disorders such as antisocial and borderline personality disorder. $^{3}$

Multiple suicide screening tools are available to clinicians, although none have proven to be clearly superior. Two common scales include the Mini-International Neuropsychiatric Interview (MINI) and SAD PERSONS scales. ${ }^{63,64}$ The Quality of Life In Epilepsy (QOLIE) survey also contains subquestions pertaining to suicidality, and can be used in the chronic care of epilepsy patients. Links to the various versions of the QOLIE can be found at http://www.rand.org/health/ surveys_tools/qolie/, and http://professionals.epilepsy.com/ page/qoltools.html. The QOLIE has not been validated for 
use as a screening tool for suicidality; however, it can serve as an ongoing metric for monitoring mental health over time in epilepsy patients.

Many screening tools for depression are available for general clinical use and could be considered in the care of epilepsy patients. One of the most common is the PHQ-9. ${ }^{65}$ In a meta-analysis review, the PHQ-9 was found to have a sensitivity of $81 \%$ and specificity of $92 \%$ for the detection of depression. ${ }^{66}$ The value of this tool in an individual patient is correlated to the a priori risk of depression in that patient. For example, if one assumes a $10 \%$ pretest probability of depression in a patient, the probability of depression is $53 \%$ with a positive screen, and $2.2 \%$ with a negative screen result. However, in a higher risk scenario with a $30 \%$ pretest probability of depression, the probability of depression with a positive screen is $81 \%$, and $8.1 \%$ with a negative result. ${ }^{65}$ The PHQ-9 takes less than 3 minutes to perform. ${ }^{67} \mathrm{PHQ}-9$ results can be followed over time, and can also serve as a monitoring tool for long-term care.

\section{Counseling epilepsy patients about the FDA AED suicidality warning}

The FDA warning creates a quandary for physicians and patients. Physicians have expressed concern that the warning may adversely affect medication compliance, leading to an increase in the morbidity, socioeconomic consequences, and potential mortality associated with uncontrolled seizures. ${ }^{54}$ One might argue that the magnitude of the absolute suicidality risk was small and as such does not merit emphasis in AED counseling. On the other hand, a statistically significant increase in suicidality risk was identified on review of aggregated data from clinical trials of 11 AEDs. Therefore, the ethics of disclosure and consent can be argued to suggest an obligation for the clinician to share these data when counseling patients regarding AED treatment. ${ }^{68}$ As an analogy, there is little controversy regarding the advisability of counseling for other rare yet potentially serious AED complications such as Stevens-Johnson syndrome and lamotrigine, ${ }^{69}$ hepatotoxicity and valproate, ${ }^{70}$ and aplastic anemia, hepatoxicity, and felbamate. ${ }^{71}$ Thus, we advocate a balanced discussion with patients regarding the FDA report recommendations, as well as the benefits of seizure control and improved quality of life that AEDs can bring. Given the aforementioned shortcomings in the diagnosis of mental illness in epilepsy patients, clinicians should also consider ways to improve the detection of psychiatric ill- ness in this population by judicious use of screening tools available.

\section{Management recommendations for health care providers}

The patient and family should be informed as to the generally increasing higher prevalence of psychiatric disorders in epilepsy, and educated as to the warning signs that may signal suicide risk. Patients can be reassured that although the rate of suicidal behavior was statistically increased with AED treatment versus placebo, the magnitude and rate was low, and for every suicide, there were 70 instances of nonfatal suicidality behaviors, which suggest the potential for prevention. Counseling regarding the consequences of nontreatment of epilepsy such as injury and SUDEP should be judiciously discussed in a patient leaning against therapy. ${ }^{72}$ Although data regarding death rates in untreated epilepsy patients are not directly available, the standardized mortality rate associated with uncontrolled versus controlled epilepsy has been determined to range from 1.6 to $9.3 .^{10}$

As stated earlier, screening for mental illness and suicidality is probably inadequate in the care of epilepsy patients, ${ }^{28}$ as in the primary care environment. ${ }^{73}$ In one study, $45 \%$ of suicide victims had contact with their primary care providers within 1 month of their death. ${ }^{74}$ Since neurologists are often a primary physician contact for epilepsy patients, this emphasizes the importance of screening for mental illness and suicidality by epilepsy physicians. Inquiry regarding the presence of comorbidities that increase the risk of suicidality should be performed in a consistent manner. Factors that increase suicide risk include alcohol and substance abuse, and concurrent psychiatric conditions such as depression, psychosis, and anxiety. In patients deemed to be at increased risk, it is appropriate to ask about a plan and whether they have access to a firearm or other methods of lethality. Urgent psychiatric assistance should be sought if significant risk is identified.

Finally, psychiatric services need to be routinely employed in the care of epilepsy patients when indicated. Although there is a small potential for seizure exacerbation associated with certain antidepressants, psychopharmacologic treatment can be successfully achieved without seizure exacerbation and should not be withheld in patients when clinically indicated..$^{9,75,76}$ Families should be made aware of the signs suggestive of suicidality, and encouraged to promptly notify their health care providers when 
present so that proper evaluation and treatment can be initiated.

\section{Conclusion}

Mental illness is a significant comorbidity in epilepsy. Suicidal behavior and successful suicides are increased in epilepsy patients compared to the general population. The role that AEDs play in this comorbidity is at present unknown. What is known and generally accepted is that successful control of seizures is associated with improved quality of life, and lack of seizure control is associated with significant morbidity and in some cases death. The 2008 FDA warning that AEDs increase suicidal behavior has rekindled attention to this clinical problem. Epileptologists and neurologists in general acknowledge that the use of some AEDs may infrequently be associated with an increased risk for suicidal behavior. However, many have criticized the FDA's decision to include all anticonvulsants in the warning, and some have questioned the validity of the meta-analysis methodology. Randomized controlled studies specifically addressing whether certain individual AEDs increase the incidence of psychiatric morbidity and suicidal behaviors are needed. In the absence of these data, we advocate routine screening of mood disorders in the epilepsy patient and a balanced discussion of the potential benefits and side effects of AED therapy, including suicidal behavior.

\section{Disclosure}

The authors report no conflicts of interest in this work.

\section{References}

1. Blisard KS, McFeeley PJ. The spectrum of neuropathologic findings in deaths associated with seizure disorders. J Forensic Sci. 1988; 33:910-914.

2. Barooni S, Thambirajah Balachandra A, Lee L. Death in epileptic people: a review of Manitoba's medical examiner's cases. J Forensic Legal Med. 2007; 14:275-278.

3. Jones JE, Hermann BP, Barry JJ, et al. Rates and risk factors for suicide, suicidal ideation, and suicide attempts in chronic epilepsy. Epilepsy Behav. 2003;4:S31-S38.

4. Nock MK, Borges G, Bromet EJ, et al. Suicide and suicidal behavior. Epidem Rev. 2008;30:133-154.

5. Karch DL, Dahlberg LL, Patel N, et al. Surveillance for violent deaths: national violent death reporting system, 16 States, 2006. MMWR Morb Mortal Wkly Rep Morbid Mort kly Rep. 2009; Surveillance Summaries. 58:1-44.

6. Prudhomme C. Epilepsy and suicide. J Nervous Mental Dis. 1941;94: 722-731.

7. Henriksen B, Juul-Jensen P, Lund M. Mortality of epileptics. In: Brackenridge R, editor. Life Assurance Medicine. London, UK: Pitman Medical 1970:139-148.

8. Bell GS, Gaitatzis A, Bell CL, et al. Suicide in people with epilepsy: how great is the risk? Epilepsia. 2009;50:1933-1942.
9. Trivedi MH, Kurian BT. Managing depressive disorders in patients with epilepsy. Psychiatry. 2007;4:26-34.

10. Hitiris N, Mohanraj R, Norrie J, et al. Mortality in epilepsy. Epilepsy Behav. 2007;10:363-376.

11. Wada K, Iwasa H, Okada M, et al. Marital status of patients with epilepsy with special reference to the influence of epileptic seizures on the patient's married life. Epilepsia. 2004;45:33-36.

12. Sillanpaa M, Haataja L, Shinnar S. Perceived impact of childhoodonset epilepsy on quality of life as an adult. Epilepsia. 2004;45: 971-977.

13. Keene DL, Loy-English I, Ventureyra EC. Long-term socioeconomic outcome following surgical intervention in the treatment of refractory epilepsy in childhood and adolescence. Child Nerv Sys. 1998;14: 362-365.

14. Carran MA, Kohler CG, O’Connor MJ, et al. Marital status after epilepsy surgery. Epilepsia. 1999;40:1755-1760.

15. The RESt-1 Group. Social aspects of epilepsy in the adult in seven European countries. The RESt-1 Group. Epilepsia. 2000;41:998-1004.

16. Kalinin VV. Suicidality and antiepileptic drugs: is there a link? Drug Saf. 2007;30:123-142.

17. Mula M, Sander JW. Negative effects of antiepileptic drugs on mood in patients with epilepsy. Drug Saf. 2007;30:555-567.

18. Jacoby A, Johnson A, Chadwick D. Psychosocial outcomes of antiepileptic drug discontinuation. The Medical Research Council Antiepileptic Drug Withdrawal Study Group. Epilepsia. 1992;33:1123-1131.

19. Kilinc S, Campbell C. The experience of discontinuing antiepileptic drug treatment: an exploratory investigation. Seizure. 2008;17:505-513.

20. Gordon K, MacSween J, Dooley J, et al. Families are content to discontinue antiepileptic drugs at different risks than their physicians. Epilepsia. 1996;37:557-562.

21. FDA. Suicidality and Antiepileptic Drugs. 2008. Available from: http:// www.fda.gov/cder/drug/infopage/antiepileptics/default.htm. Accessed on Jun 10, 2010.

22. Hauser WA, Annegers JF, Rocca WA. Descriptive epidemiology of epilepsy: contributions of population-based studies from Rochester, Minnesota. Mayo Clin Proceed. 1996;71:576-586.

23. Kwan P, Brodie MJ. Early identification of refractory epilepsy. N Engl JMed. 2000;342:314-319.

24. Lawn ND, Bamlet WR, Radhakrishnan K, et al. Injuries due to seizures in persons with epilepsy: a population-based study. Am Acad Neurol. 2004;63: 1565-1570.

25. Noe KH, Drazkowski JF. Safety of long-term video-electroencephalographic monitoring for evaluation of epilepsy. Mayo Clin Proceed. 2009;84:495-500.

26. Tellez-Zenteno JF, Ronquillo LH, Wiebe S. Sudden unexpected death in epilepsy: evidence-based analysis of incidence and risk factors. Epilepsy Res. 2005;65:101-115.

27. Sperling MR, Feldman H, Kinman J, et al. Seizure control and mortality in epilepsy. Ann Neurol. 1999;46:45-50.

28. Gilliam FG, Santos J, Vahle V, et al. Depression in epilepsy: ignoring clinical expression of neuronal network dysfunction? Epilepsia. 2004; 45:28-33.

29. Boro A, Haut S. Medical comorbidities in the treatment of epilepsy. Epilepsy Behav. 2003;4:S2-S12.

30. Tellez-Zenteno JF, Patten SB, Jette N, et al. Psychiatric comorbidity in epilepsy: a population-based analysis. Epilepsia. 2007;48:2336-2344.

31. Schmitz B. Depression and mania in patients with epilepsy. Epilepsia. 2005;46:45-49.

32. Shneker BF, Cios JS, Elliott JO. Suicidality, depression screening, and antiepileptic drugs: reaction to the FDA alert. Neurology. 2009;72: 987-991.

33. Dodrill CB, Batzel LW. Interictal behavioral features of patients with epilepsy. Epilepsia. 1986;27:S64-S76.

34. Kanner AM. Epilepsy, suicidal behaviour, and depression: do they share common pathogenic mechanisms? Lancet Neurol. 2006;5:107-108. 
35. Mendez MF, Doss RC, Taylor JL, et al. Depression in epilepsy: relationship to seizures and anticonvulsant therapy. J Nerv Mental Dis. 1993;181:444-447.

36. Brent DA, Crumrine PK, Varma RR, et al. Phenobarbital treatment and major depressive disorder in children with epilepsy. Pediatrics. 1987; 80:909.

37. Trimble MR, Rüsch N, Betts T, et al. Psychiatric symptoms after therapy with new antiepileptic drugs: psychopathological and seizure related variables. Seizure. 2000;9:249-254.

38. Mula M, Sander JW. Suicidal ideation in epilepsy and levetiracetam therapy. Epilepsy Behav. 2007;11:130-132.

39. Jacoby A, Baker GA, Steen N, et al. The clinical course of epilepsy and its psychosocial correlates: findings from a UK community study. Epilepsia. 1996;37:148-161.

40. O'Donoghue MF, Goodridge DM, Redhead K, et al. Assessing the psychosocial consequences of epilepsy: a community-based study. $\mathrm{BrJ}$ Gen Pract. 1999;49:211-214.

41. Mula M, Trimble MR. The importance of being seizure free: topiramate and psychopathology in epilepsy. Epilepsy Behav. 2003;4: 430-434.

42. Nilsson L, Tomson T, Farahmand BY, et al. Cause-specific mortality in epilepsy: a cohort study of more than 9,000 patients once hospitalized for epilepsy. Epilepsia. 1997;38:1062-1068.

43. Rafnsson V, Olafsson E, Hauser WA, et al. Cause-specific mortality in adults with unprovoked seizures. A population-based incidence cohort study. Neuroepidemiol. 2001;20:232-236.

44. Hauser WA, Annegers JF, Elveback LR. Mortality in patients with epilepsy. Epilepsia. 1980;21:399-412.

45. Lhatoo SD, Johnson AL, Goodridge DM, et al. Mortality in epilepsy in the first 11 to 14 years after diagnosis: Multivariate analysis of a long-term, prospective, population-based cohort. Ann Neurol. 2001;49: 336-344.

46. Nilsson L, Ahlbom A, Farahmand BY, et al. Risk factors for suicide in epilepsy: a case control study. Epilepsia. 2002;43:644-651.

47. Christensen J, Vestergaard M, Mortensen PB, et al. Epilepsy and risk of suicide: a population-based case-control study. Lancet Neurol. 2007;6:693-698.

48. Mainio A, Alamaki K, Karvonen K, et al. Depression and suicide in epileptic victims: a population-based study of suicide victims during the years 1988-2002 in northern Finland. Epilepsy Behav. 2007;11:389-393.

49. Kalinin VV, Polyanskiy DA. Gender and suicidality prediction in epilepsy. Epilepsy Behav. 2005;7:657-663.

50. Fukuchi T, Kanemoto K, Kato M, et al. Death in epilepsy with special attention to suicide cases. Epilepsy Res. 2002;51:233-236.

51. Hesdorffer DC, Hauser WA, Olafsson E, et al. Depression and suicide attempt as risk factors for incident unprovoked seizures. Ann Neurol. 2006;59:35-41.

52. Avorn J. Drug warnings that can cause fits - communicating risks in a data-poor environment. $N$ Engl J Med. 2008;359:991-994.

53. Levenson M, Mentari E, Hughes A, et al. Statistical review and evaluation: antiepileptic drugs and suicidality. In: Research CfDEa, editor.: Department of Health and Human Services 2008:1-45.

54. Hesdorffer DC, Kanner AM. The FDA alert on suicidality and antiepileptic drugs: fire or false alarm? Epilepsia. 2009;50:978-986.

55. Kanner AM, Cole AJ. Comorbid depressive disorders in epilepsy. CNS Spectr. 2010;15:3-6.
56. Bell GS, Mula M, Sander JW. Suicidality in people taking antiepileptic drugs: what is the evidence? CNS Drugs. 2009;23:281-292.

57. Olesen JB, Hansen PR, Erdal J, et al. Antiepileptic drugs and risk of suicide: a nationwide study. Pharmacoepidemiol Drug Safety. 2010;19: 518-524.

58. Patorno E, Bohn RL, Wahl PM, et al. Anticonvulsant medications and the risk of suicide, attempted suicide, or violent death. JAMA. 2010;303: 1401-1409.

59. Gibbons RD, Hur K, Brown CH, et al. Relationship between antiepileptic drugs and suicide attempts in patients with bipolar disorder. Arch Gen Psychiatry. 2009;66:1354-1360.

60. van Cott AC, Cramer JA, Copeland LA, et al. Suicide-related behaviors in older patients with new anti-epileptic drug use: data from the VA hospital system. BMC Med. 2010;8:1-7.

61. Andersohn F, Schade R, Willich SN, et al. Use of antiepileptic drugs in epilepsy and the risk of self-harm or suicidal behavior. Neurology. 2010; 75:335-340

62. Porkorny A. Prediction of suicide in psychiatric patients: report of a prospective study. Arch Gen Psychiatry. 1983;40:249-257.

63. Sheehan DV, Lecrubier Y, Sheehan KH, et al. The validity of the Mini International Neuropsychiatric Interview (MINI) according to the SCID-P and its reliability. Eur Psychiatry. 1997;12:232-241.

64. Hockberger RS, Rothstein RJ. Assessment of suicide potential by nonpsychiatrists using the sad persons score. JEmergency Med. 1988;6: 99-107.

65. Ebell MH. Screening instruments for depression. Am Fam Physician. 2008;78:244-246.

66. Gilbody S, Richards D, Brealey S, et al. Screening for depression in medical settings with the Patient Health Questionnaire (PHQ): a diagnostic meta-analysis. J Gen Int Med. 2007;22:1596-1602.

67. Spitzer RL, Kroenke K, Williams JBW. Patient health questionaire study group. Validity and utility of a self-report version of PRIME-MD: the PHQ primary care study. JAMA. 1999;282:1737-1744.

68. Lo B. Informed consent. In Lo B, editor. Resolving Ethical Dilemmas: A guide for clinicians. 3rd ed. Philadelphia, PA: Lippincott Williams \& Wilkins 2005

69. Guberman AH, Besag FM, Brodie MJ, et al. Lamotrigine-associated rash: risk/benefit considerations in adults and children. Epilepsia. 1999; 40:985-991

70. Harden CL. Therapeutic safety monitoring: what to look for and when to look for it. Epilepsia. 2000;41:S37-S44.

71. Pellock JM. Felbamate in epilepsy therapy: evaluating the risks. Drug Saf. 1999;21:225-239.

72. Nilsson L, Ahlbom A, Farahmand BY, et al. Mortality in a populationbased cohort of epilepsy surgery patients. Epilepsia. 2003;44:575-581.

73. Hepner KA, Rowe M, Rost K, et al. The effect of adherence to practice guidelines on depression outcomes. Ann Intern Med. 2007;147: 320-329.

74. Luoma JB, Martin CE, Pearson JL. Contact with mental health and primary care providers before suicide: a review of the evidence. $A m J$ Psychiatry. 2002;159:909-916.

75. Harden CL, Goldstein MA. Mood disorders in patients with epilepsy: epidemiology and management. CNS Drugs. 2002;16:291-302.

76. Prueter C, Norra C. Mood disorders and their treatment in patients with epilepsy. J Neuropsych Clin Neurosci. 2005;17:20-28.

Drug, Healthcare and Patient Safety

\section{Publish your work in this journal}

Drug, Healthcare and Patient Safety is an international, peer-reviewed open-access journal exploring patient safety issues in the healthcare continuum from diagnostic and screening interventions through to treatment, drug therapy and surgery. The journal is characterized by the rapic reporting of reviews, original research, clinical, epidemiological and

post-marketing surveillance studies, risk management, health literacy and educational programs across all areas of healthcare delivery. The manuscript management system is completely online and includes a very quick and fair peer-review system. Visit http://www.dovepress.com/ testimonials.php to read real quotes from published authors. 